\title{
Effects of robot-assisted gait training with active motion visual feedback induced by guidance force on walking speed in patients with chronic stroke: a pilot study
}

\author{
DOI: https://doi.org/10.5114/pq.2020.95776
}

\author{
Mu-Hong Yoon ${ }^{1}$, Duck-Won Oh \\ ${ }^{1}$ Department of Physical Therapy, Immanuel Medical Rehabilitation \& Long-Term Care Hospital, Graduate School, \\ Cheongju University, Cheongju, Republic of Korea \\ ${ }^{2}$ Department of Physical Therapy, College of Health Science, Cheongju University, Cheongju, Republic of Korea
}

\begin{abstract}
Introduction. This study aimed to determine the effects of robot-assisted gait training with active movement visual feedback induced by guidance force on walking speed in patients with chronic stroke.

Methods. A single-subject reversal (A-B) design was applied. Overall, 3 patients with chronic stroke underwent robot-assisted gait training with visual feedback displaying active motion of the affected lower limb during the intervention phase. Walking function was measured by using the 10-m walk test (10MWT) and peak knee flexion angle (PKFA) during walking.

Results. During the intervention phase, the 10MWT score of subjects 1, 2, and 3 improved by $23.95 \%, 30.95 \%$, and $43.71 \%$, respectively, and the PKFA improved by $8.41 \%, 15.92 \%$, and $32.25 \%$, respectively. The walking speed and PKFA in all subjects after the training showed improvement when compared with the baseline phase $(p<0.05)$.

Conclusions. These findings suggest that robot-assisted gait training with active motion visual feedback and guidance force may be clinically helpful to improve walking recovery after stroke.

Key words: robot-assisted gait training, feedback, walking, stroke
\end{abstract}

\section{Introduction}

After a stroke, sensory-motor impairments may be a major cause of asymmetrical walking patterns and decreased walking speed. Therefore, the major goals in stroke rehabilitation include regaining the ability to walk independently and to carry out routine activities as soon as possible [1]. At present, neurological gait rehabilitation techniques to improve walking function have been mostly based on physical therapy interventions, including neurophysiological and motor learning concepts [2]. All approaches involve clinician observation, specifically designed preparative exercises, and therapistguided movement of the lower limbs during walking, followed by overground walking training [3]. However, research has shown that most patients with chronic stroke do not recover a normal gait pattern following conventional gait training. Early intervention usually involves conservative rehabilitation training, and current clinical practices have combined it with robotic assistance [4].

Current evidence suggests that high-intensity repetitive task-specific practice may be the most beneficial method to restore functional performance after stroke. Robot-assisted gait training is a technique used worldwide in physical therapy that applies this method to promote the walking function of patients after stroke [5]. It may be valuable in saving therapists' effort and time by assuring consistent movement and safety during post-stroke walking training [6]. However, the basic motion of walking is provided by the robotic system, so that the legs of patients with chronic stroke are forced to passively move in a serial motion of walking. This reduces the physical effort of patients with chronic stroke and potentially interferes with the learning of walking skills [7]. Therefore, it is important to make the patients more active, particularly encouraging them to actively start the walking motion by reducing the amount of help provided by the robotic walking system [8].

In terms of recent robotic assistance strategies, the assist-as-needed control concept has advanced from facilitating active motion and participation to training patients after stroke, with the aim to either assist in or correct their movements [2]. In the robotic system, the guidance force can be set in order to control the force that assists with the movement of the hip and knee joints [8]. Furthermore, visual feedback presenting body movements, given by guidance force, constitutes an important aspect of the patients' active effort by increasing motivation and facilitating participation in the training process [9]. One of the advantages of robot-assisted gait training is to offer feedback on the training, which is a necessary process in learning functional skills and developing problem-solving abilities related to movement [10].

The input of visual information has a positive effect on motor control during walking by forming a cognitive process related to action in the central nervous system and integrating it with other sensory information [11]. In addition, given that it is capable of directly identifying functional outcomes during training, visual feedback training may be helpful to guide patients with stroke to facilitate repeated training and learning in their own way [12]. On the basis of this concept, recent studies have supported the use of visual feedback to improve balance and walking function of patients with chronic stroke [13]. However, to the best of our knowledge, little attention has been paid to studying the effects of visual feed-

Correspondence address: Duck-Won Oh, Department of Physical Therapy, College of Health Science, Cheongju University, 298 Daeseong-ro, Cheongwon-gu, Cheongju, Chungcheongbuk-do, Republic of Korea, 28503, e-mail: odduck@cju.ac.kr 
back presenting active movement derived from guidance force during robot-assisted gait training. Thus, the purpose of this study was to demonstrate the effects of robot-assisted gait training with active movement visual feedback and guidance force to enhance walking function in patients with chronic stroke.

\section{Subjects and methods}

\section{Subjects}

Three patients with chronic stroke who were able to independently perform daily activities volunteered for this study. The selection criteria were as follows: (1) stroke onset $>6$ months before; (2) independent walking with and without walking aids; (3) no orthopaedic, cardiopulmonary, or neurologic diseases except stroke; (4) mild spasticity of the affected leg ( $£$ G2 on the modified Ashworth scale); and (5) no cognitive impairment (> 24 points on the Korean version of the Mini-Mental State Examination) [14]. Patients with stroke-related symptoms that impeded training, such as serious sensory impairment, aphasia, or hemispatial neglect, were excluded from the study. A detailed explanation about the experimental process and safety was provided to all subjects prior to the training. Table 1 describes the clinical characteristics of the subjects.

\section{Study design}

A single-subject reversal (A-B) design was applied in the study. During the baseline and intervention phases, measurements were performed in 8 sessions each (16 sessions total). Robot-assisted gait training with active motion visual feedback and guidance force was only performed during the intervention phase, and measurements were performed immediately after the training. Additionally, the patients underwent a daily 40-minute therapy routine during each phase, including mat exercises and therapist-guided activities (sit-to-stand, stand alone, and step forwards and backwards).

\section{Outcome measures}

10-m walk test (10MWT)

In the 10MWT, the time taken to walk the middle 10-m range of a 14-m path at a comfortable walking speed was measured to exclude the acceleration and deceleration periods of walking. The 10MWT has been reported to have a high intra- and inter-rater reliability $(r: 0.89-1.00)$ [15]. Values were averaged over 3 trials with a 1-minute rest interval.

\section{Peak knee flexion angle (PKFA)}

The PKFA was measured by recording a video clip of the affected side while the patient performed the 10MWT. To effectively evaluate the PKFA, markers were attached to the greater trochanter, fibular head, and lateral malleolus of the affected side [16]. The peak height of the affected knee in the swing phase during the 10MWT was captured with a video capture program (VapMix, Vapshion, Korea) and the PKFA was analysed with the ImageJ software (ImageJ, National Institutes of Health, USA). Along with the 10MWT, the PKFA values were also averaged over 3 trials.

\section{Robot-assisted gait training}

Robot-assisted gait training was performed with a robotic system (Lokomat ${ }^{\circledR}$, Hocoma AG, Zurich, Switzerland), consisting of a display monitor, a robotic exoskeleton, a weight support device, and a treadmill (Woodway $\mathrm{GmbH}$, Weil am Rhein, Germany) (Figure 1). The initial step was to attach the exoskeleton of the robotic system to the patient's legs after adjusting the length, and to fasten the weight support device in the patient standing on the treadmill to ensure safety during the training process. Additionally, a foot lift was worn to prevent toe dragging in the swing phase. Subsequently, the system was operated to guide the flexion and extension motions of the hip and knee joints. For all subjects, the walking speed of the treadmill was initially set at $1.3 \mathrm{~km} / \mathrm{h}$, with an increase of $0.1 \mathrm{~km} / \mathrm{h}$ every 10 minutes for a 30 -minute period. The extent of body weight support was started at $50 \%$ and

Table 1. Clinical details of the subjects

\begin{tabular}{|c|c|c|c|}
\hline Variables & Subject 1 & Subject 2 & Subject 3 \\
\hline Age (years) & 52 & 50 & 65 \\
\hline Gender & Female & Female & Female \\
\hline Affected side & Left & Left & Right \\
\hline Duration (months) & 7 & 10 & 8 \\
\hline Type of stroke & Haemorrhage & Haemorrhage & Haemorrhage \\
\hline MMSE-K (score) & 28 & 30 & 28 \\
\hline MAS & G1 & G1 & $\mathrm{G} 1+$ \\
\hline $\mathrm{MBI}$ & 75 & 61 & 59 \\
\hline Fugl-Meyer Assessment (sensory) & 18 & 17 & 19 \\
\hline \multicolumn{4}{|l|}{ Stroke outcome classification } \\
\hline Number of impaired neurological domains & 1 & 1 & 1 \\
\hline Severity of impairment & A & A & B \\
\hline Function & I & II & II \\
\hline
\end{tabular}




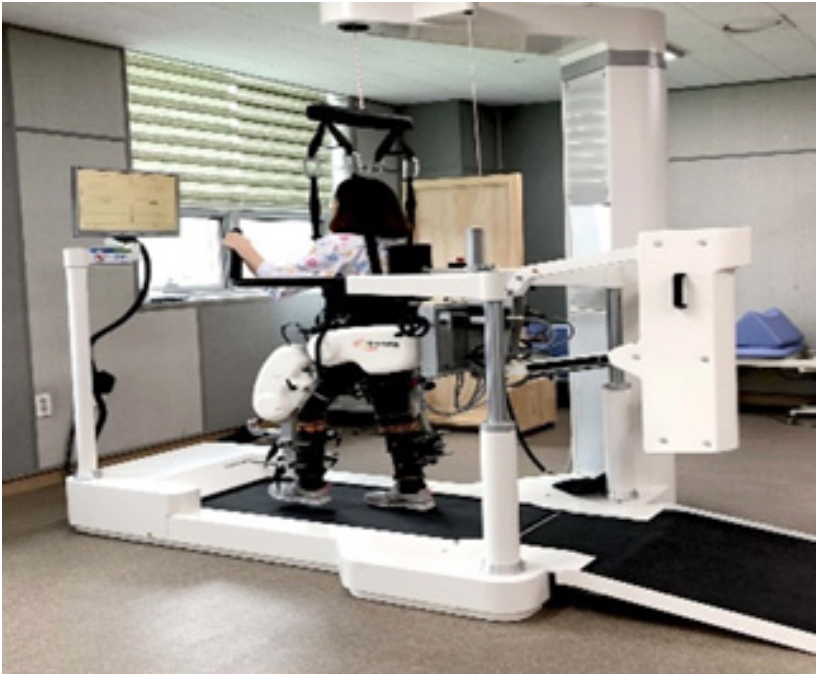

Figure 1. Robot-assisted gait training

was decreased by $10 \%$ every 10 minutes. During all intervention sessions, the training followed this procedure to monitor the progress in speed and weight relief.

The guidance force was set to facilitate the active participation of the subjects during the training. It was adjusted in accordance with a synchronization value, which indicates the consistency between the movement speed of the robotic system provided by the subject's effort and the treadmill speed. Subjects 1, 2, and 3 started at $100 \%$ of guidance force (indicating the maximum assistance), which was gradually decreased up to $60 \%, 70 \%$, and $85 \%$, respectively, depending on each patient's synchronization value.

Visual feedback reflected the active motion of hip and knee joints of the affected leg to maintain the synchronization between the robotic system and treadmill movements. It was displayed as bar graphs dynamically changing on the front monitor. As visual feedback, the graph of active motion represents the sensor responses to the subtle changes of force adaptation in the system and provides the degree of mutual force between the patient and the robotic system. Active motion visual feedback encourages the patient to be more active, and the sensors in the robotic system continue to react and adjust the graph. In the final 5 minutes of the training, the visual feedback was removed to facilitate the patient's effort to control the active motion of the legs.

\section{Data analysis}

The statistical analysis was performed by using the SPSS 22.0 software for Windows. In autocorrelation analysis, data collected during the baseline phase did not show significant serial dependency $(p<0.05)$. The walking speed and PKFA determined at each measurement session of the baseline and intervention phases were expressed as mean and standard deviation, and they were shown as a dotted line graph for visual analysis. Additionally, the 2-standard-deviation (2SD) band method was used to increase the reliability of the visual analysis and confirm the clinical significance with $\geq 2$ data points in the intervention phase over the $2 S D$ band of the baseline phase [17]. Furthermore, a paired $t$-test served to compare the walking speed and PKFA between the baseline and intervention phases in each subject. Statistical significance was set at $p<0.05$. In these variables, the effect size associated with intervention was calculated with the standardized mean difference method [18].

\section{Ethical approval}

The research related to human use has complied with all the relevant national regulations and institutional policies and has followed the tenets of the Declaration of Helsinki.

\section{Informed consent}

Informed consent has been obtained from all individuals included in this study.

\section{Results}

Table 2 and Figures 2 and 3 summarize the walking speed and PKFA measured during the baseline and intervention phases in each subject. During the intervention phase, the walking speed of subjects 1, 2, and 3 improved by $23.95 \%$, $30.95 \%$, and $43.71 \%$, respectively, and the PKFA improved by $8.41 \%, 15.92 \%$, and $32.25 \%$, respectively. Moreover, in the $2 S D$ band analysis, a clinically significant improvement was found in all subjects with respect to walking speed, and for subjects 2 and 3 with respect to PKFA. All participants showed a statistically significant improvement of the values of walking speed and PKFA between the baseline phase and the intervention phase $(p<0.05)$. Furthermore, the effect sizes related to the intervention were very small for $10 \mathrm{MWT}$ $(d=0.39)$ and large for PKFA $(d=1.01)[19]$.

\section{Discussion}

The presented study suggests that robot-assisted gait training with active motion visual feedback induced by guidance force may be favourably used to improve the walking speed of patients with chronic stroke.

In the study, we applied visual feedback presenting active motion by setting the guidance force during robot-assisted gait training to induce the interest of patients with chronic stroke and promote motor performance [20]. Although mechanical support and motion control in robotic systems provides an opportunity to repeatedly practise walking skills in a safe way, it seems to be somewhat difficult to induce muscle activities of the legs in patients with chronic stroke because the basic element of robot-assisted gait training is to make repetitively passive walking motions [6]. In robot-assisted gait training, the current strategy has adopted the assist-as-needed control concept to encourage active motion of the patients by assisting in or correcting leg movement during walking [2]. Therefore, as used in this study, active motion visual feedback induced by guidance force may be considered a beneficial means of promoting the participation of patients with chronic stroke in robot-assisted gait training [8].

The main result of this study is that robot-assisted gait training with visual feedback presenting the active motion of the leg helps improve the walking speed in patients with stroke. Active movement and participation are needed to resolve movement errors by experiencing a variety of movement patterns, contributing to neural plasticity in the central nervous system [8]. Therefore, a possible explanation for our findings is that the integration of active motion into the training may help develop various movement patterns [9] and facilitate the learning of walking skills by practising them in conditions similar to the real environment [21]. Furthermore, previous research has demonstrated the importance of active intervention in stroke rehabilitation, which is also necessary to maintain motivation and training adherence for improved effects [22].

In this study, guidance force was adjusted to display visual feedback of active motion during the robot-assisted 

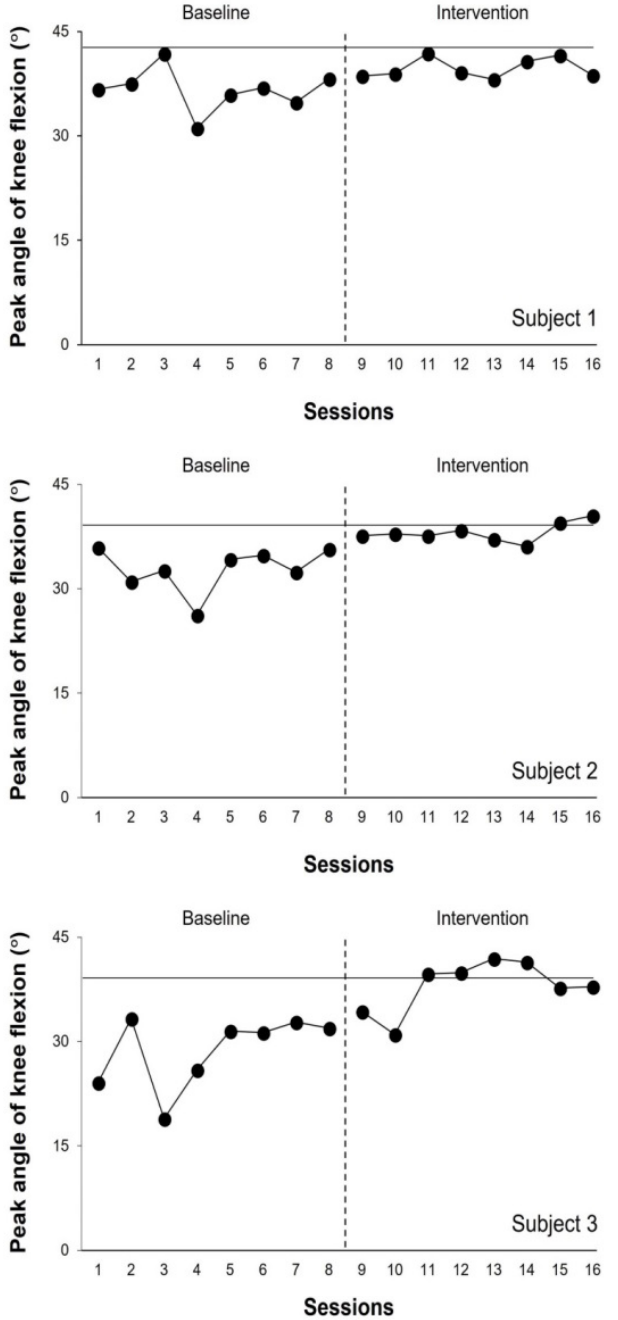

Figure 2. Changes in walking speed of each subject during the baseline and intervention phases. The horizontal line across the phases indicates the upper limit of the $2 S D$ band of the baseline phase
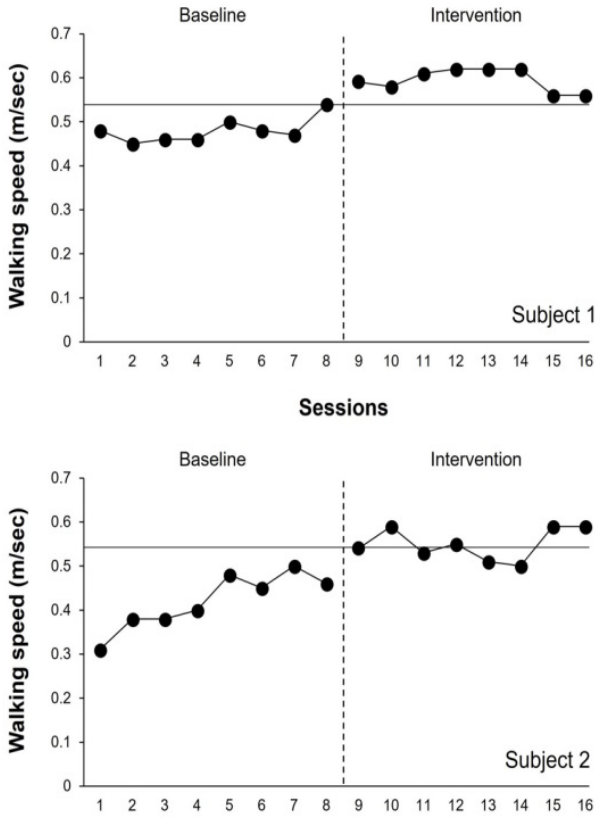

Sessions

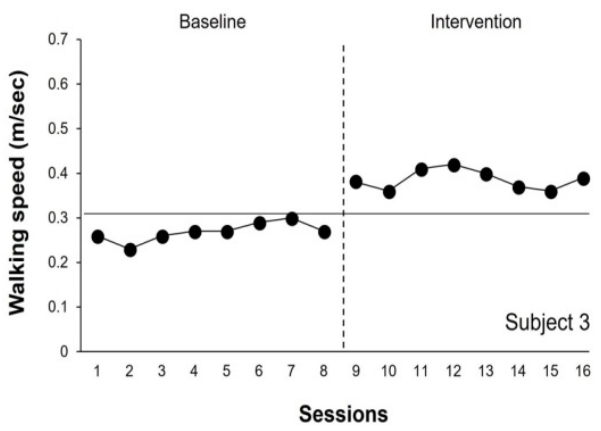

Figure 3. Changes in peak angle of knee flexion of each subject during the baseline and intervention phases. The horizontal line across the phases indicates the upper limit of the $2 S D$ band of the baseline phase

Table 2. Comparison of walking speed and PKFA measured during the baseline and intervention phases in each subject

\begin{tabular}{|c|c|c|c|}
\hline Parameters & Subject 1 & Subject 2 & Subject 3 \\
\hline \multicolumn{4}{|l|}{ 10MWT (m/s) } \\
\hline Baseline phase & $0.48 \pm 0.28$ & $0.42 \pm 0.06$ & $0.26 \pm 0.02$ \\
\hline Intervention phase & $0.59 \pm 0.26$ & $0.55 \pm 0.03$ & $0.38 \pm 0.02$ \\
\hline$t(p)$ & $-7.33(0.00)$ & $-5.19(0.00)$ & $-10.40(0.00)$ \\
\hline \multicolumn{4}{|l|}{ PKFA $\left({ }^{\circ}\right)$} \\
\hline Baseline phase & $36.61 \pm 3.03$ & $32.85 \pm 3.18$ & $28.71 \pm 5.18$ \\
\hline Intervention phase & $39.69 \pm 1.46$ & $38.08 \pm 1.38$ & $37.97 \pm 3.70$ \\
\hline$t(p)$ & $-2.99(0.02)$ & $-4.17(0.00)$ & $-3.86(0.01)$ \\
\hline
\end{tabular}

10MWT - 10-m walk test, PKFA - peak knee flexion angle

training. In general, a greater guidance force indicates more assistance to maintain the walking pattern during the training; therefore, it has a negative impact on motor learning because of decreased active participation during the training process. Thus, as shown in this study, gradually decreasing the guidance force during the training may be a good option for improved effects of robot-assisted gait training. Recent studies have shown that reducing the guidance force during robot-assisted gait training improves cortical activity associated with sensory-motor information processing [23] and helps to increase muscle activity [8]. Using visual feedback based on guidance force, robot-assisted gait training may be an effective way to encourage active participation of patients with chronic stroke during the training. Previous studies have also reported that visual feedback training is helpful in restoring post-stroke function [24]. 
The PKFA determined in the study increased during the intervention phase. Walking requires repetitive flexion and extension movements of each joint in the legs to produce the propulsive force of the leg [25]. The knee joint absorbs the reaction forces from the ground in the initial stance, and also contributes to the advancement of the leg by flexing during the swing phase [26]. By such reason, hip and knee flexion ranges noticeably add to walking velocity [27]. However, patients with chronic stroke present circumduction motion of the affected leg to avoid toe dragging caused by reduced knee flexion during the swing phase. Consequently, this compensatory walking pattern has been considered to decrease walking speed [28]. Therefore, as seen in this study, increased PKFA contributes to the improvement of walking speed. Similar to our results, a previous study has also supported the use of robot-assisted gait training to increase muscle activity and walking speed after stroke [6].

\section{Limitations}

The study has several limitations that can be corrected in future studies. First, we used a single-subject experimental research design to investigate the effects of our intervention. Although this design has some advantages for investigators with a limited research environment by systematically studying individual responses of the subjects to the intervention [29], it may be difficult to generalize our findings beyond our sample group. Second, only the short-term effects of the intervention were measured; therefore, our results cannot be used to interpret long-term results of the intervention. Finally, the study did not include quantitative measurements to identify gait parameters. Future studies with a larger sample size and longer follow-up period are necessary to clarify our results.

\section{Conclusions}

In stroke rehabilitation, robotic systems have some advantages for post-stroke walking recovery, which include increased motivation by offering visual feedback on movements, as well as the facilitation of active motion of legs by setting the guidance force. This study was performed to determine whether robot-assisted gait training with visual feedback presenting active motion of legs on the basis of guidance force improved the walking speed in patients with chronic stroke. The results showed that such training might be helpful to increase the walking speed of patients with chronic stroke. Further studies with robust design are needed to provide more definite findings.

\section{Disclosure statement}

No author has any financial interest or received any financial benefit from this research.

\section{Conflict of interest}

The authors state no conflict of interest.

\section{References}

1. Lynch EA, Hillier SL, Stiller K, Campanella RR, Fisher PH. Sensory retraining of the lower limb after acute stroke: a randomized controlled pilot trial. Arch Phys Med Rehabil. 2007;88(9):1101-1107; doi: 10.1016/j.apmr.2007. 06.010 .

2. Belda-Lois J-M, Mena-del Horno S, Bermejo-Bosch I, Moreno JC, Pons JL, Farina D, et al. Rehabilitation of gait after stroke: a review towards a top-down approach. J
Neuroeng Rehabil. 2011;8:66; doi: 10.1186/1743-00038-66.

3. Pollock A, Baer G, Pomeroy V, Langhorne P. Physiotherapy treatment approaches for the recovery of postural control and lower limb function following stroke. Cochrane Database Syst Rev. 2007;1:CD001920; doi: 10.1002/14651858.CD001920.pub2.

4. Tedla JS, Dixit S, Gular K, Abohashrh M. Robotic-assisted gait training effect on function and gait speed in subacute and chronic stroke population: a systematic review and meta-analysis of randomized controlled trials. Eur Neurol. 2019;81(3-4):103-111; doi: 10.1159/0005 00747.

5. Langhorne $P$, Coupar F, Pollock $A$. Motor recovery after stroke: a systematic review. Lancet Neurol. 2009;8(8): 741-754; doi: 10.1016/S1474-4422(09)70150-4.

6. Husemann B, Müller F, Krewer C, Heller S, Koenig E. Effects of locomotion training with assistance of a robotdriven gait orthosis in hemiparetic patients after stroke: a randomized controlled pilot study. Stroke. 2007;38(2): 349-354; doi: 10.1161/01.STR.0000254607.48765.cb.

7. Mirelman A, Bonato P, Deutsch JE. Effects of training with a robot-virtual reality system compared with a robot alone on the gait of individuals after stroke. Stroke. 2009; 40(1):169-174; doi: 10.1161/STROKEAHA.108.516328.

8. Duschau-Wicke A, von Zitzewitz J, Caprez A, Lunenburger L, Riener R. Path control: a method for patient-cooperative robot-aided gait rehabilitation. IEEE Trans Neural Syst Rehabil Eng. 2010;18(1):38-48; doi: 10.1109/TNSRE.2009.2033061.

9. Marchal-Crespo L, Reinkensmeyer DJ. Review of control strategies for robotic movement training after neurologic injury. J Neuroeng Rehabil. 2009;6:20; doi: 10.1186/ 1743-0003-6-20.

10. Lünenburger L, Colombo G, Riener R. Biofeedback for robotic gait rehabilitation. J Neuroeng Rehabil. 2007;4: 1; doi: 10.1186/1743-0003-4-1.

11. Bonan IV, Yelnik AP, Colle FM, Michaud C, Normand E, Panigot $B$, et al. Reliance on visual information after stroke. Part II: Effectiveness of a balance rehabilitation program with visual cue deprivation after stroke: a randomized controlled trial. Arch Phys Med Rehabil. 2004; 85(2):274-278; doi: 10.1016/j.apmr.2003.06.016.

12. Rizzo AA, Bowerly T, Buckwalter JG, Klimchuk D, Mitura $\mathrm{R}$, Parsons TD. A virtual reality scenario for all seasons: the virtual classroom. CNS Spectr. 2006;11(1):35-44; doi: $10.1017 /$ S1092852900024196.

13. Drużbicki M, Guzik A, Przysada G, Kwolek A, Brzozowska-Magoń A. Efficacy of gait training using a treadmill with and without visual biofeedback in patients after stroke: a randomized study. J Rehabil Med. 2015;47(5): 419-425; doi: 10.2340/16501977-1949.

14. Folstein MF, Folstein SE, McHugh PR. "Mini-mental state": a practical method for grading the cognitive state of patients for the clinician. J Psychiatr Res. 1975;12(3):189198; doi: 10.1016/0022-3956(75)90026-6.

15. Steffen TM, Hacker TA, Mollinger L. Age- and genderrelated test performance in community-dwelling elderly people: Six-Minute Walk Test, Berg Balance Scale, Timed Up \& Go Test, and gait speeds. Phys Ther. 2002;82(2): 128-137; doi: 10.1093/ptj/82.2.128.

16. Norkin CC, White DJ. Measurement of joint motion: a guide to goniometry. Philadelphia: FA Davis; 2016.

17. Miller EW, Combs SA, Fish C, Bense B, Owens A, Burch A. Running training after stroke: a single-subject 
report. Phys Ther. 2008;88(4):511-522; doi: 10.2522/ ptj.20050240.

18. Olive ML, Smith BW. Effect size calculations and single subject designs. Educ Psychol. 2005;25(2-3):313-324; doi: 10.1080/0144341042000301238.

19. Sawilowsky SS. New effect size rules of thumb. J Mod Appl Stat Methods. 2009;8(2):597-599; doi: 10.22237/ jmasm/1257035100.

20. Ahn M-H, Park K-D, You Y-Y. The effect of feedback on somesthetic video game training for improving balance of stroke patients. J Korean Soc Phys Med. 2012;7(4):401409; doi: 10.13066/kspm.2012.7.4.401.

21. Israel JF, Campbell DD, Kahn JH, Hornby TG. Metabolic costs and muscle activity patterns during robotic- and therapist-assisted treadmill walking in individuals with incomplete spinal cord injury. Phys Ther. 2006;86(11): 1466-1478; doi: 10.2522/ptj.20050266.

22. Park YS, Kweon SS. Factors affecting stroke patients' rehabilitation motivation. Korean Public Health Res. 2002; 28(1):21-30.

23. Knaepen K, Mierau A, Swinnen E, Tellez HF, Michielsen M, Kerckhofs E, et al. Human-robot interaction: does robotic guidance force affect gait-related brain dynamics during robot-assisted treadmill walking? PLoS One. 2015; 10(10):e0140626; doi: 10.1371/journal.pone.0140626.

24. Quaney BM, He J, Timberlake G, Dodd K, Carr C. Visuomotor training improves stroke-related ipsilesional upper extremity impairments. Neurorehabil Neural Repair. 2010; 24(1):52-61; doi: 10.1177/1545968309341646.

25. Coutts F. Gait analysis in the therapeutic environment. Man Ther. 1999;4(1):2-10; doi: 10.1016/s1356-689x(99) 80003-4.

26. Decker MJ, Torry MR, Wyland DJ, Sterett WI, Steadman JR. Gender differences in lower extremity kinematics, kinetics and energy absorption during landing. Clin Biomech. 2003;18(7):662-669; doi: 10.1016/S0268-0033 (03)00090-1.

27. Escalante A, Lichtenstein MJ, Hazuda HP. Walking velocity in aged persons: its association with lower extremity joint range of motion. Arthrit Care Res. 2001;45(3):287294; doi: 10.1002/1529-0131(200106)45:3<287::AIDART262>3.0.CO;2-1.

28. Sutherland DH, Olshen R, Cooper L, Woo SL. The development of mature gait. J Bone Joint Surg Am. 1980;62(3): 336-353; doi: 10.2106/00004623-198062030-00004.

29. Portney LG, Watkins MP. Foundations of clinical research: applications to practice. Upper Saddle River: Prentice Hall; 2015. 